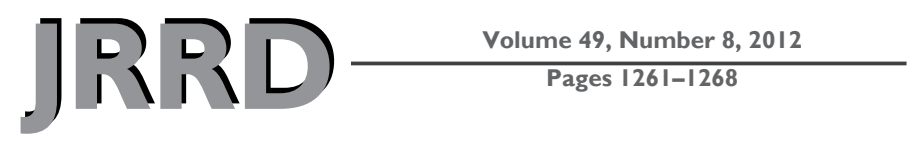

\title{
Activity monitor accuracy in persons using canes
}

\author{
Deborah Michael Wendland, PT, DPT, CPed; ${ }^{1^{*}}$ Stephen H. Sprigle, PhD, PT ${ }^{1-2}$ \\ ${ }^{1}$ School of Applied Physiology and ${ }^{2}$ School of Industrial Design, Georgia Institute of Technology, Atlanta, GA
}

\begin{abstract}
The StepWatch activity monitor has not been validated on multiple indoor and outdoor surfaces in a population using ambulation aids. The aims of this technical report are to report on strategies to configure the StepWatch activity monitor on subjects using a cane and to report the accuracy of both leg-mounted and cane-mounted StepWatch devices on people ambulating over different surfaces while using a cane. Sixteen subjects aged 67 to $85 \mathrm{yr}$ (mean 75.6) who regularly use a cane for ambulation participated. StepWatch calibration was performed by adjusting sensitivity and cadence. Following calibration optimization, accuracy was tested on both the legmounted and cane-mounted devices on different surfaces, including linoleum, sidewalk, grass, ramp, and stairs. The legmounted device had an accuracy of $93.4 \%$ across all surfaces, while the cane-mounted device had an aggregate accuracy of $84.7 \%$ across all surfaces. Accuracy of the StepWatch on the stairs was significantly less accurate $(p<0.001)$ when comparing surfaces using repeated measures analysis of variance. When monitoring community mobility, placement of a StepWatch on a person and his/her ambulation aid can accurately document both activity and device use.
\end{abstract}

Key words: accelerometry, accuracy, ambulation aids, assistive device, canes, elderly, gait, mobility limitation, physical activity monitoring, walking.

\section{INTRODUCTION}

Physical activity is important for health and has been associated with a decrease in all-cause mortality [1-3]. Additionally, physical activity has been connected to health benefits including improved cardiorespiratory fitness, metabolic health, weight maintenance, functional health, cancer risk reduction, and improved mental health [4].
Furthermore, according to a report from the Urban Institute of the U.S. Department of Health and Human Services, there has been an increased prevalence of assistive device use to facilitate increased independence with activities of daily living such as mobility and instrumental activities of daily living [5]. Simple equipment such as walkers, canes, and crutches showed the largest growth in usage [5]. While research offers some indication that assistive devices may replace human help in some cases, the effect of this is unknown [5].

Given the increase in assistive device use and the value of physical activity, it is important to recognize that activity varies across populations [6-7]. With such variation, it is also essential to acknowledge that facilitation of physical activity must reflect these differences. One useful means to promote and measure activity is with an activity monitor that, by measuring activity, can help to grade activity changes [8] as well as motivate people to be active [9].

The StepWatch (Orthocare Innovations; Mountlake Terrace, Washington) is one example of a commonly used accelerometer-based monitor that reflects physical activity by reporting strides during ambulation. It has been utilized and/or validated in a large number of populations, including the elderly [10-12] and those with

\footnotetext{
Abbreviations: ANOVA = analysis of variance, $\mathrm{SCI}=$ spinal cord injury.

*Address all correspondence to Deborah Michael Wendland, PT, DPT, CPed; 3001 Mercer University Dr, Davis Building, 100A, Atlanta, GA 30341-4155; 678-547-6775; fax: 678-547-6202. Email: wendland dm@mercer.edu http://dx.doi.org/10.1682/JRRD.2011.08.0141
} 
spinal cord injury (SCI) [13-14], amputation [15], stroke [16-18], muscular dystrophy [19], and diabetes mellitus [20-22]. Some people who use mobility assistive devices have been studied in the context of other, larger studies using StepWatch [13-14,16-17,23-24].

Bowden and Behrman used StepWatch to monitor ambulation in 11 persons with incomplete SCI, 9 of whom used an ambulation device [13]. For those using ambulation aids, the overall stride accuracy of the 10-meter walk was 97 percent, with individual stride accuracy ranging between 85 and 100 percent [13].

In a study of activity in persons after stroke, Haeuber et al. studied 17 people, 15 of whom used an ambulation device [17]. StepWatch accuracy was measured using 1 min bouts of walking at self-selected and faster speeds. For all subjects, the StepWatch reported a 94 percent accuracy using the default configuration settings of cadence $=80$ and sensitivity $=12$ [17]. Both of these studies conclude that the StepWatch is accurate enough to monitor the activity of persons using ambulation aids indoors.

In a different study looking at gait in people after a stroke, Mudge et al. described the subjects as being able to "walk independently" with their customary orthotic devices, but no mention was given to any ambulatory aid [23]. While this study utilized different surfaces, there was no clear use of ambulation aids. In a population that uses ambulation aids such as canes, documenting gait accuracy of the monitoring device on different surfaces consistent with everyday mobility would be beneficial because gait patterns often change while ambulating over outdoor surfaces, including ramps and uneven terrain [25-27]. These surface changes and subsequent gait pattern modifications might affect StepWatch accuracy as well. Moreover, documenting the accuracy of a StepWatch mounted to a cane can be beneficial to studies investigating the use of ambulation aids during everyday mobility.

The aims of this technical report were to report on (1) strategies to individualize StepWatch configuration parameters for leg and cane-mounted monitors, (2) the accuracy of the StepWatch as persons ambulate over different surfaces and grades while using a cane, and (3) StepWatch accuracy in measuring cane-ground contact as a practical means of documenting cane usage.

\section{METHODS}

\section{Participants}

People who used canes during ambulation were recruited from Senior Centers and Senior Housing in metropolitan Atlanta, Georgia. To be included in the study, subjects had to be over 18 years old, use an assistive device for gait, have the ability to ambulate at least $10 \mathrm{~m}$ without rest, and be able to give informed consent.

\section{Instrumentation}

StepWatch is a U.S. Food and Drug Administrationcleared class II device designed for long-term assessment of community walking function. It is $75 \times 50 \times 20 \mathrm{~mm}$ and weighs $38 \mathrm{~g}$. StepWatch output consists of stride counts over a user-defined epoch. Two parameters, cadence and sensitivity, can be adjusted for individuals. Cadence limits the rate at which the StepWatch detects steps. As cadence settings are raised, the device will increase the time delay before the next stride can be detected. Therefore, higher cadences should be chosen for slow walkers and for people with long legs [28]. Sensitivity, on the other hand, is a measure of the amount of movement required by the StepWatch before it detects a step. As the sensitivity settings are raised, the device requires more movement to record a step [28].

\section{Protocol}

Subjects were fitted with a StepWatch device. The device was placed on each subject's right ankle just proximal to his or her lateral malleolus using a Velcro band. A second StepWatch device was placed on the distal end of his or her regular cane using double-sided tape and porous tape to further secure the device. No adjustments were made to either the subjects' canes or their gait patterns.

The protocol included two stages (Figure 1). In the first stage, the StepWatch parameters were evaluated and optimized using short bouts of mobility. Leg-monitor accuracy was defined by comparing the number of strides recorded by StepWatch with the number of strides observed by a researcher. Strides were manually counted each time the right leg had forward progression. Canemounted accuracy was defined by comparing the number of "strides" recorded by StepWatch to the number of times that the cane made contact with the ground as observed by a researcher. Separate researchers used 


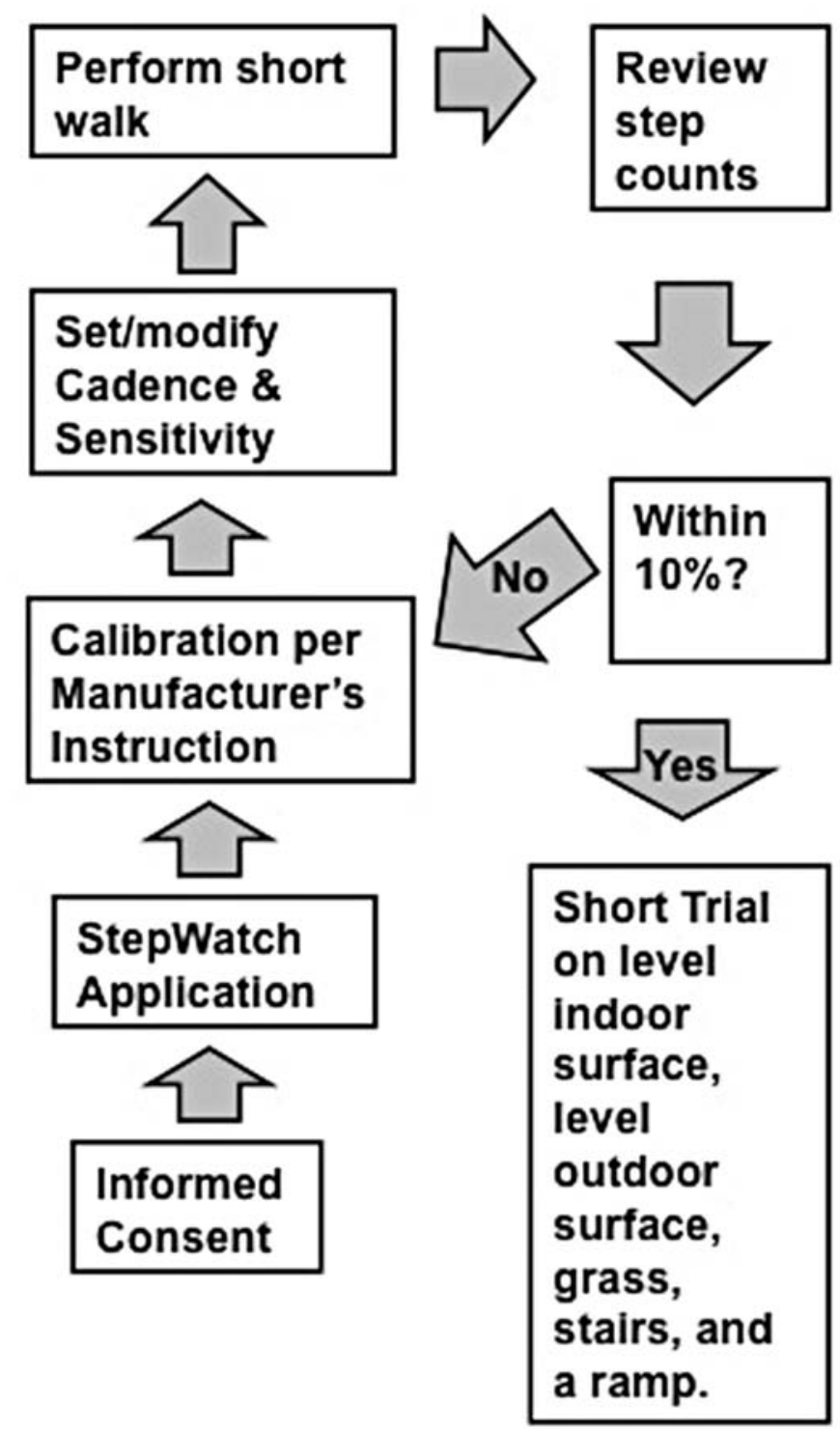

Figure 1.

Two-stage study protocol.

handheld counters (GOGO, Atafa; Cambridge, Massachusetts) to document strides and cane-ground contacts for comparison with the recorded data from the leg- and cane-mounted StepWatch devices, respectively. In the second stage, bouts of ambulation were taken over different surfaces and grades. Leg strides and cane-ground contacts were manually counted by separate researchers to calculate accuracy.

For stage 1, both the leg-mounted and cane-mounted StepWatch devices were initially configured according to the manufacturer's specification. The cadence setting was defined using the individual's height, and the suggested sensitivity setting relative to each device's calibrated threshold was initially used [28]. These numbers were then input into the advanced programming setting along with a 6 s epoch time.

Calibration trial ambulation was performed in a room such that each subject ambulated in one direction, around a table in a circle, at a given speed. The subject then turned around and ambulated in the opposite direction at a slower speed. Both self-selected and slower speeds were used to calculate accuracy. The total distance covered with each calibration trial was typically between 30 and 50 strides. Acceptable accuracy for both devices was defined when the recorded strides were within 10 percent of the manually counted strides and cane-ground contacts.

Parameters for the StepWatch were modified in a manner similar to Bowden and Behrman [13]. If the StepWatch overcounted strides, sensitivity was raised an increment. When the monitor undercounted during the trial, sensitivity was lowered an increment. Normally, to minimize subject fatigue, if the device's recorded strides were 10 or more counts different than the counted strides or cane-ground contacts, sensitivity was increased or decreased by two increments rather than one. This process was repeated until StepWatch values were within 10 percent of the manual count. Figure 1 illustrates the calibration procedure.

Once calibrated, the StepWatch devices were remounted to each participant's right leg and cane. Each subject then proceeded with the validation protocol. The validation protocol required that participants ambulate over an indoor level surface (linoleum), an outdoor level surface (sidewalk), an outdoor uneven surface (grass), up and down a ramp, and up and down stairs. Two $10 \mathrm{~m}$ distances were completed for each surface. At least $30 \mathrm{~s}$ of rest was given between bouts. Leg strides and caneground contacts were manually counted by separate researchers using the same handheld tally counters. The indoor and outdoor level surfaces were always completed first to allow for an assessment of the participant's safety during ambulation. Following those trials, the remaining three tasks were performed in a randomly assigned order to minimize the effect of fatigue on the gait pattern.

Upon completion of the protocol, the StepWatch monitors were removed and the data were downloaded per the manufacturer's specification. StepWatch software was used to process data and report the number of strides taken during each bout of activity. Data were analyzed using 
PASW Statistics software (IBM; Armonk, New York). Descriptive data analysis was performed comparing the manual counts with the recorded StepWatch data for both stages of this study. A repeated measures analysis of variance (ANOVA) with post hoc pairwise comparison tests was used to compare accuracy across surfaces and device location. Huynh-Feldt was used to correct for sphericity. A Bonferroni correction was used to correct for multiple comparisons. Finally, a $t$-test was used to compare the accuracy between the leg- and cane-mounted StepWatch devices. Alpha level was set at $\alpha<0.05$.

\section{RESULTS}

Sixteen subjects ranging in age from 67 to $85 \mathrm{yr}$ (mean 75.6) met qualification criteria and agreed to participate. On level surfaces, every subject ambulated with a 2-point gait pattern using his or her straight cane. In all but one case, cadence settings were effectively set for both leg- and cane-mounted monitoring devices according to the person's height without any adjustment. The subject with an ineffective height-based cadence setting exhibited an antalgic gait pattern, which changed from trial to trial. With this individual, we were unable to calibrate either StepWatch device. That subject did not complete the study.

Sensitivity setting adjustments were required to calibrate the leg-mounted StepWatch monitors in only seven of the subjects completing the study (Table). Five of these subjects required lowering the sensitivity setting, while two required that the sensitivity setting be raised. Six of the seven subjects requiring sensitivity modification needed an adjustment of one unit from the default value. In the remaining case, the subject required a two unit adjustment from the default setting.

Calibration of the cane-mounted StepWatch devices required sensitivity setting adjustment in all but one of the 15 subjects completing the study (Table). The sensitivity setting had to be lowered for the cane-mounted monitors in 12 of these cases. The remaining two subjects needing modification required the sensitivity setting be raised. Of the 14 requiring adjustment to the sensitivity setting, 8 required a lowering of the sensitivity by three or four units and 1 required a decrease in the sensitivity setting by two units.

During stage 1 of the protocol, the calibrated legmounted StepWatch monitors had 98 percent accuracy,
Table.

Sensitivity calibration for leg- and cane-mounted StepWatch devices.

\begin{tabular}{lll}
\hline Subject & $\begin{array}{c}\text { Leg-Mounted } \\
\text { Sensitivity } \\
\text { Modification }\end{array}$ & $\begin{array}{c}\text { Cane-Mounted } \\
\text { Sensitivity } \\
\text { Modification }\end{array}$ \\
\hline 1 & $\downarrow 1$ unit & $\downarrow 3$ units \\
2 & No adjustment & $\downarrow 3$ units \\
3 & No adjustment & $\downarrow 4$ units \\
4 & $\uparrow 1$ unit & $\downarrow 3$ units \\
5 & $\uparrow 1$ unit & $\downarrow 1$ unit \\
6 & No adjustment & $\downarrow 4$ units \\
7 & No adjustment & $\downarrow 3$ units \\
8 & Unable to calibrate & Unable to calibrate \\
9 & $\downarrow 1$ unit & $\downarrow 4$ units \\
10 & No adjustment & $\uparrow 1$ unit \\
11 & No adjustment & $\downarrow 3$ units \\
12 & $\downarrow 2$ units & $\uparrow 1$ unit \\
13 & $\downarrow 1$ unit & No adjustment \\
14 & No adjustment & $\downarrow 2$ units \\
15 & No adjustment & $\downarrow 1$ unit \\
16 & $\downarrow 1$ unit & $\downarrow 1$ unit \\
\hline \hline
\end{tabular}

while the calibrated cane-mounted monitors had 97 percent accuracy.

For stage 2 of the protocol, aggregated manual and recorded counts over the entire trial, both indoor and outdoor, resulted in an accuracy measure for the legmounted device of 93.4 percent. The cane-mounted device recorded an aggregate accuracy of 84.7 percent. These values were significantly different when compared using a $t$-test $(p<0.001)$.

Specific surfaces were found to have different levels of accuracy for the leg-mounted and the cane-mounted monitors (Figure 2). When the surfaces were compared using the repeated measures ANOVA, there was a main effect $(p<0.001)$. With post hoc pairwise comparisons, the accuracy on the stair surface was significantly lower than all the other surfaces $(p<0.01)$. There was no interaction between surface and device. If stairs were excluded in the aggregation, leg-mounted accuracy was improved to 95.8 percent and cane-mounted accuracy was improved to 89.1 percent.

On the whole, the cane-mounted device had lower accuracies than the leg-mounted monitor. The lowest accuracies for both leg- and cane-mounted StepWatch monitors were recorded during ambulation on stairs $(85.9 \% \pm 10.4 \%$ and $64.0 \% \pm 25.7 \%$, respectively). The surfaces with the highest accuracy for the cane-mounted StepWatch were the 


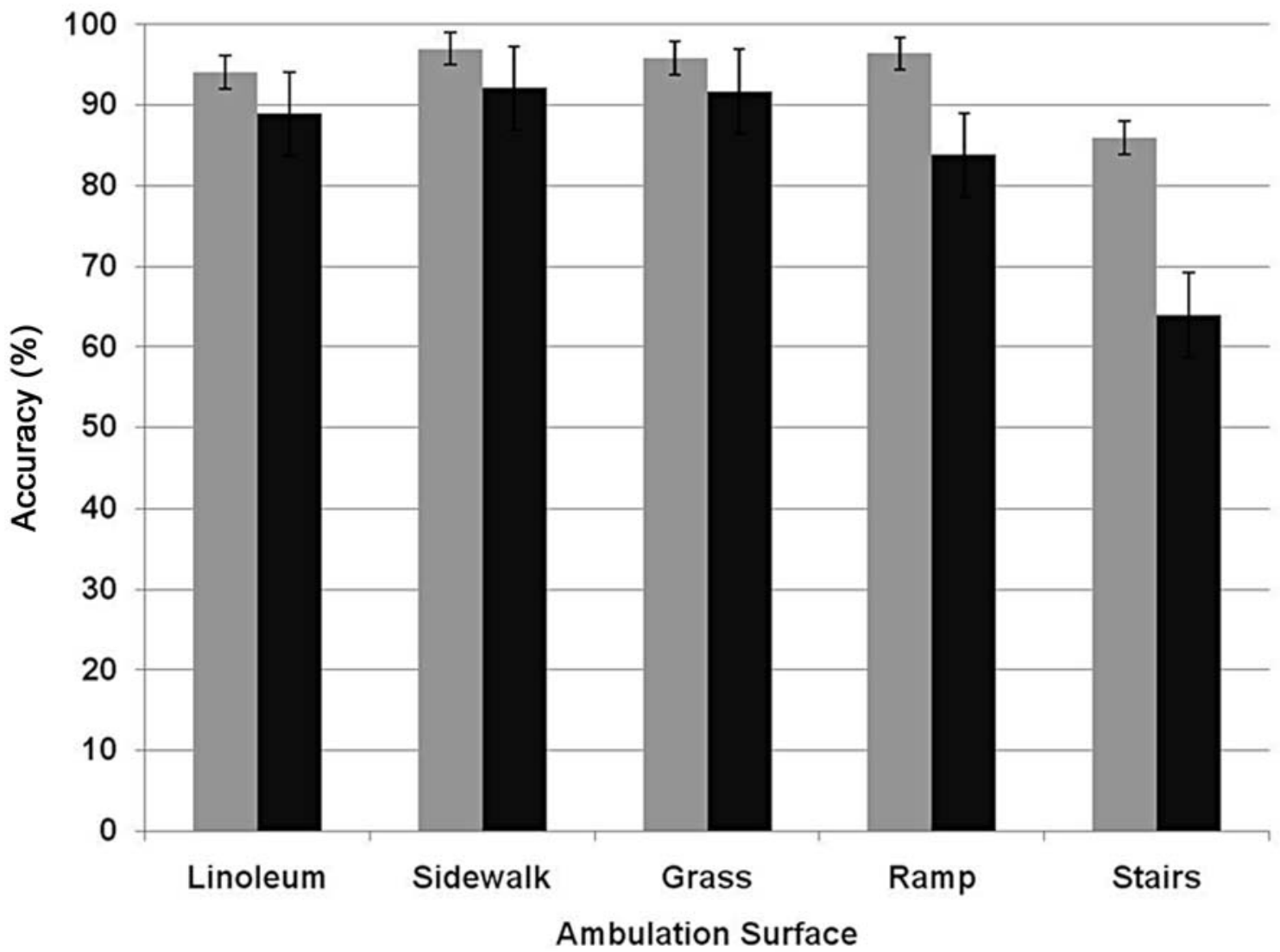

Figure 2.

Accuracy of StepWatch during validation trial compared with manually counted leg strides and cane-ground contacts. Stair values were significantly different from other surfaces when compared using pairwise comparison post hoc for repeated measures analysis of variance $(p<0.01, \alpha<0.05$ with Bonferroni correction). Bars indicate standard error of mean.

sidewalk $(92.1 \% \pm 7.9 \%)$ and the grass $(91.7 \% \pm 4.7 \%)$. Accuracy of the StepWatch on the leg for the same surfaces was $97.0 \pm 2.8$ percent (sidewalk), and $95.8 \pm 3.7$ percent (grass) (Figure 2). Aggregated accuracy was affected by individual surface accuracy as well as strides recorded between trials.

\section{DISCUSSION}

We found that short ambulation bouts can be used to optimize cadence and sensitivity settings for both leg- and cane-mounted StepWatch devices. Ease of calibration was affected by the limited stamina of this elderly cohort, so settings should be optimized using as little iteration as possible. For leg-mounted units, a judicious approach is to begin with the settings as recommended by the manufac- turer. For cane-mounted devices, we recommend starting the calibration process slightly differently. The cadence setting may be based on the person's height, but the starting point for sensitivity calibration may be adjusted to two units below the default setting to increase calibration efficiency. Alternatively, "jumps" of two sensitivity units could be made when the trial ambulation bouts report a mismatch between recorded and observed scores. Tight turns, leg movement, cane tapping, and cane carrying affected StepWatch accuracy on the cane-mounted device in particular. In addition, we found that an antalgic gait can be prohibitive to accurately calibrate leg- and canemounted StepWatch devices.

For cane users, we found that the leg-mounted StepWatch accurately recorded the number of strides for ambulation over various surfaces and grades with a combined accuracy of 93.4 percent. The surface accuracy 
ranged from 86 percent on stairs to 97 percent on the sidewalk. The StepWatch device was more accurate on nonstair surfaces for the leg-mounted device. Given that people spend little time walking on stairs, the legmounted device accuracy will allow clinicians to use the StepWatch in this population with confidence that everyday mobility will be appropriately reflected in longerterm trials.

The cane-mounted StepWatch had an aggregate accuracy across all surfaces of 85 percent. The specific surface accuracy ranged from 64 percent on stairs to 92 percent on the sidewalk. Accuracy was improved when stairs were excluded (89.1\%). This is likely related to the variability with which a cane is utilized. During ambulation on level surfaces, participants consistently utilized a 2-point gait pattern, which the StepWatch monitored accurately. While traversing stairs, participants used a nonreciprocal step-to pattern while gripping a railing, which altered the need for supplementary balance assistance from the cane. Based on visual feedback, the decreased accuracy resulted from the tendency of the subjects to use the cane differently, including carrying the cane or cane tapping.

In certain populations, monitoring cane usage can be helpful to the immediate health of the individual. For example, people with diabetes with plantar ulceration should ambulate in a way that the ulcer is off-loaded and often includes the use of an assistive device. By closely monitoring the use of an assistive device in conjunction with leg-monitoring, further injury from an excessive increase of physical stress (steps) [29] can be avoided [8]. Additionally, spikes in physical activity levels have been associated with an increased risk of skin breakdown [30]. Potentially, using an activity monitor for feedback may facilitate consistency in physical activity levels, which could reduce the risk to the integumentary system.

Feedback indicated that the StepWatch can be used on a cane with little effect on the user's daily life. Since the person never has to interact with the StepWatch, compliance is not an issue and its measurements therefore reflect the utilization of the cane.

Limitations of this study are primarily due to the cohort studied and the resulting variability of how they utilized a cane. Cane behaviors like tapping or carrying may be measured with an activity monitor even though they may not constitute a true step. Additionally, tight turns will affect the distance that canes are moved, thus affecting whether strides are actually recorded or not. In addition, gait patterns that change secondary to pain, for instance, may be prohibitively difficult to calibrate. This will limit the generalizability of the findings to that component of this population.

Also, accuracy for both the leg- and cane-mounted StepWatch devices may also be biased lower secondary to the surface protocol used for stage 2 of this study. Because participants used a step-to pattern on stairs, the number of strides required to complete the $10 \mathrm{~m}$ level surface walks was approximately half the number required to complete the stair task. With the disproportionate strides taken on the stairs, the increased weighting of stair surface accuracy depressed overall accuracy. Because the prevalence of traversing stairs in real life is minimal compared with negotiating level surfaces, we believe the cane-mounted StepWatch accurately reflects cane usage.

For future work, an analogous StepWatch validation could be completed on other assistive devices, such as standard walkers and crutches, which are used by various populations with mobility disabilities. With further validation, the StepWatch could be used to monitor ambulation of persons using an array of assistive devices in order to study the everyday activities of persons with disabilities.

\section{CONCLUSIONS}

Calibration of StepWatch monitors can be performed according to manufacturer's instructions for both legand cane-mounted devices in a population that uses ambulation aids. When monitoring community mobility, placement of a StepWatch on a person and his or her ambulation aid can accurately document both activity and device use.

\section{ACKNOWLEDGMENTS}

\section{Author Contributions:}

Study concept and design: D. M. Wendland, S. H. Sprigle. Acquisition of data: D. M. Wendland.

Analysis and interpretation of data: D. M. Wendland, S. H. Sprigle.

Drafting and critical revision of manuscript: D. M. Wendland,

S. H. Sprigle.

Statistical Analysis: D. M. Wendland, S. H. Sprigle.

Study supervision: S. H. Sprigle.

Financial Disclosures: The authors have declared that no competing interests exist. 
Funding/Support: This material was based on work supported by the National Institutes of Health (grant T32HD055180).

Additional Contributions: We would like to thank Susan Perlman for her assistance with data collection. D. M. Wendland is now with the Department of Physical Therapy in the College of Pharmacy and Health Sciences at Mercer University, Atlanta, Georgia.

Institutional Review: All subjects signed an informed consent approved by the Georgia Institute of Technology Institution Review Board prior to participation in the study.

Participant Follow-Up: The authors have no plans to notify the study subjects of the publication of this article because of a lack of contact information.

\section{REFERENCES}

1. Paganini-Hill A, Kawas CH, Corrada MM. Activities and mortality in the elderly: the Leisure World cohort study. J Gerontol A Biol Sci Med Sci. 2011;66(5):559-67. [PMID:21350247] http://dx.doi.org/10.1093/gerona/glq237

2. Woodcock J, Franco OH, Orsini N, Roberts I. Non-vigorous physical activity and all-cause mortality: systematic review and meta-analysis of cohort studies. Int J Epidemiol. 2011;40(1):121-38. [PMID:20630992] http://dx.doi.org/10.1093/ije/dyq104

3. Lee DC, Sui X, Ortega FB, Kim YS, Church TS, Winett RA, Ekelund U, Katzmarzyk PT, Blair SN. Comparisons of leisure-time physical activity and cardiorespiratory fitness as predictors of all-cause mortality in men and women. $\mathrm{Br}$ J Sports Med. 2011;45(6):504-10. [PMID:20418526] http://dx.doi.org/10.1136/bjsm.2009.066209

4. Physical Activity Guidelines Advisory Committee. Physical activity guidelines advisory committee report. Washington (DC): U.S. Department of Health and Human Services; 2008.

5. Spillman BC. Assistive device use among the elderly: trends, characteristics of users, and implications for modeling executive summary. Washington (DC): U.S. Department of Health and Human Services; 2005.

6. Bohannon RW. Number of pedometer-assessed steps taken per day by adults: a descriptive meta-analysis. Phys Ther. 2007;87(12):1642-50. [PMID:17911274]

http://dx.doi.org/10.2522/ptj.20060037

7. Tudor-Locke C, Washington TL, Hart TL. Expected values for steps/day in special populations. Prev Med. 2009;49(1): 3-11. [PMID:19409409] http://dx.doi.org/10.1016/j.ypmed.2009.04.012

8. Lemaster JW, Mueller MJ, Reiber GE, Mehr DR, Madsen RW, Conn VS. Effect of weight-bearing activity on foot ulcer incidence in people with diabetic peripheral neuropathy: feet first randomized controlled trial. Phys Ther. 2008;
88(11):1385-98. [PMID:18801859]

http://dx.doi.org/10.2522/ptj.20080019

9. de Bruin ED, Hartmann A, Uebelhart D, Murer K, Zijlstra W. Wearable systems for monitoring mobility-related activities in older people: a systematic review. Clin Rehabil. 2008;22(10-11):878-95. [PMID:18955420] http://dx.doi.org/10.1177/0269215508090675

10. Resnick B, Nahm ES, Orwig D, Zimmerman SS, Magaziner J. Measurement of activity in older adults: reliability and validity of the Step Activity Monitor. J Nurs Meas. 2001;9(3):275-90. [PMID:11881269]

11. Bergman RJ, Bassett DR Jr, Muthukrishnan S, Klein DA. Validity of 2 devices for measuring steps taken by older adults in assisted-living facilities. J Phys Act Health. 2008; 5(Suppl 1):S166-75. [PMID:18364521]

12. Algase DL, Beattie ER, Leitsch SA, Beel-Bates CA. Biomechanical activity devices to index wandering behavior in dementia. Am J Alzheimers Dis Other Demen. 2003;18(2): 85-92. [PMID:12708223] http://dx.doi.org/10.1177/153331750301800202

13. Bowden MG, Behrman AL. Step Activity Monitor: accuracy and test-retest reliability in persons with incomplete spinal cord injury. J Rehabil Res Dev. 2007;44(3):355-62. [PMID:18247232] http://dx.doi.org/10.1682/JRRD.2006.03.0033

14. Saraf P, Rafferty MR, Moore JL, Kahn JH, Hendron K, Leech K, Hornby TG. Daily stepping in individuals with motor incomplete spinal cord injury. Phys Ther. 2010; 90(2):224-35. [PMID:20022997] http://dx.doi.org/10.2522/ptj.20090064

15. Coleman KL, Smith DG, Boone DA, Joseph AW, del Aguila MA. Step activity monitor: long-term, continuous recording of ambulatory function. J Rehabil Res Dev. 1999;36(1):8-18. [PMID:10659890]

16. Macko RF, Haeuber E, Shaughnessy M, Coleman KL, Boone DA, Smith GV, Silver KH. Microprocessor-based ambulatory activity monitoring in stroke patients. Med Sci Sports Exerc. 2002;34(3):394-99. [PMID:11880800] http://dx.doi.org/10.1097/00005768-200203000-00002

17. Haeuber E, Shaughnessy M, Forrester LW, Coleman KL, Macko RF. Accelerometer monitoring of home- and community-based ambulatory activity after stroke. Arch Phys Med Rehabil. 2004;85(12):1997-2001. [PMID:15605339] http://dx.doi.org/10.1016/j.apmr.2003.11.035

18. Mudge S, Stott NS. Test-retest reliability of the StepWatch Activity Monitor outputs in individuals with chronic stroke. Clin Rehabil. 2008;22(10-11):871-77. [PMID:18955419] http://dx.doi.org/10.1177/0269215508092822

19. McDonald CM, Widman LM, Walsh DD, Walsh SA, Abresch RT. Use of step activity monitoring for continuous physical activity assessment in boys with Duchenne muscular dystrophy. Arch Phys Med Rehabil. 2005;86(4):802-8. 
JRRD, Volume 49, Number 8, 2012

\section{[PMID:15827935]}

http://dx.doi.org/10.1016/j.apmr.2004.10.012

20. Maluf KS, Mueller MJ. Novel Award 2002. Comparison of physical activity and cumulative plantar tissue stress among subjects with and without diabetes mellitus and a history of recurrent plantar ulcers. Clin Biomech (Bristol, Avon). 2003;18(7):567-75. [PMID:12880704] http://dx.doi.org/10.1016/S0268-0033(03)00118-9

21. Smith DG, Domholdt E, Coleman KL, Del Aguila MA, Boone DA. Ambulatory activity in men with diabetes: relationship between self-reported and real-world performance-based measures. J Rehabil Res Dev. 2004;41(4): 571-80. [PMID:15558385]

22. Kanade RV, van Deursen RW, Harding K, Price P. Walking performance in people with diabetic neuropathy: benefits and threats. Diabetologia. 2006;49(8):1747-54.

[PMID:16758177] http://dx.doi.org/10.1007/s00125-006-0309-1

23. Mudge S, Stott NS, Walt SE. Criterion validity of the StepWatch Activity Monitor as a measure of walking activity in patients after stroke. Arch Phys Med Rehabil. 2007;88(12): 1710-15. [PMID:18047890] http://dx.doi.org/10.1016/j.apmr.2007.07.039

24. Michael KM, Allen JK, Macko RF. Reduced ambulatory activity after stroke: the role of balance, gait, and cardiovascular fitness. Arch Phys Med Rehabil. 2005;86(8): 1552-56. [PMID:16084807] http://dx.doi.org/10.1016/j.apmr.2004.12.026

25. Prentice SD, Hasler EN, Groves JJ, Frank JS. Locomotor adaptations for changes in the slope of the walking surface. Gait Posture. 2004;20(3):255-65. [PMID:15531172] http://dx.doi.org/10.1016/j.gaitpost.2003.09.006
26. Redfern MS, DiPasquale J. Biomechanics of descending ramps. Gait Posture. 1997;6(2):119-25. http://dx.doi.org/10.1016/S0966-6362(97)01117-X

27. Menant JC, Steele JR, Menz HB, Munro BJ, Lord SR. Effects of walking surfaces and footwear on temporo-spatial gait parameters in young and older people. Gait Posture. 2009;29(3):392-97. [PMID:19041245] http://dx.doi.org/10.1016/j.gaitpost.2008.10.057

28. Orthocare Innovations. StepWatch 3.1 user's manual. Oklahoma City (OK): Orthocare Innovations; 2008. p. 25-26.

29. Mueller MJ, Maluf KS. Tissue adaptation to physical stress: a proposed "Physical Stress Theory" to guide physical therapist practice, education, and research. Phys Ther. 2002;82(4):383-403. [PMID:11922854]

30. Armstrong DG, Lavery LA, Holtz-Neiderer K, Mohler MJ, Wendel CS, Nixon BP, Boulton AJ. Variability in activity may precede diabetic foot ulceration. Diabetes Care. 2004; 27(8):1980-84. [PMID:15277427] http://dx.doi.org/10.2337/diacare.27.8.1980

Submitted for publication August 8, 2011. Accepted in revised form January 19, 2012.

This article and any supplementary material should be cited as follows:

Wendland DM, Sprigle SH. Activity monitor accuracy in persons using canes. J Rehabil Res Dev. 2012;49(8): 1261-68.

http://dx.doi.org/10.1682/JRRD.2011.08.0141

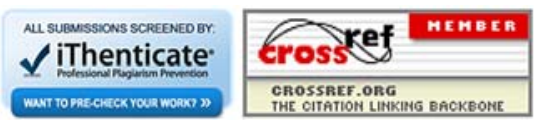

